\title{
UM MODELO PARA RECOMENDAÇÃO DE ARTIGOS ACADÊMICOS BASEADO EM FILTRAGEM COLABORATIVA APLICADO À AMBIENTES MÓVEIS
}

\author{
Sílvio César Cazella \\ Irismar Corrêa das Chagas \\ Jorge Luis V. Barbosa \\ Universidade do Vale do Rio dos Sinos \\ Av. Unisinos 950, CEP 93.022-000, São Leopoldo, RS, Brazil \\ \{cazella, jbarbosa\}@unisinos.br, irismarcorrea@gmail.com \\ Eliseo Berni Reategui \\ Universidade Federal do Rio Grande do Sul \\ Av. Paulo Gama 110, prédio 12105, Porto Alegre, RS, Brazil \\ eliseoreategui@gmail.com
}

Resumo. Sistemas de Recomendação (SR) são hoje amplamente utilizados, principalmente na área de e-commerce como, por exemplo, Amazon.com e Submarino.com. Neste trabalho, propõe-se a utilização destes sistemas na educação, especificamente para a recomendação de conteúdo em dispositivos móveis. A vantagem de se utilizar sistemas de recomendação em dispositivos móveis está na facilidade da entrega da recomendação aos usuários. Com base neste cenário, este artigo apresenta um modelo de Sistema de Recomendação baseado em Filtragem Colaborativa (FC), também conhecida como Filtragem Social (FS), para ambientes móveis. O modelo proposto foi implementado em um protótipo visando a recomendação de artigos científicos. A avaliação das recomendações recebidas foi realizada pelos usuários utilizando uma escala likert de 5 pontos. No final deste artigo são descritos alguns experimentos realizados e os resultados obtidos com a aplicação do modelo proposto são discutidos.

Palavras-chaves:filtragem colaborativa, sistemas de recomendação, computação móvel.

\section{A MODEL FOR RECOMMENDATION OF ACADEMIC PAPERS BASED ON COLLABORATIVE FILTERING APPLIED TO MOBIL ENVIRONMENTS}

\begin{abstract}
Recommender Systems (SR) are widely used nowadays specially in e-commerce sites, for example, Amazon.com and Submarino.com. In this project, we propose the use of such systems in the area of education, specifically for the recommendation of contents in mobile devices. The advantage of using Recommender Systems in mobile devices is that it is an easy way to deliver recommendations to the users. Based on this scenario, this paper presents a model of a Recommender System based on Collaborative filtering (CF) - known as Social Filtering $(S F)$ too- for mobile environments. The proposed model was implemented in a prototype aimed to recommend academic papers through mobile devices. The evaluation of the received recommendations by users was conducted using a likert scale of 5 points. At the end of this paper some experiments are described and the results obtained with the implementation of the proposed model are discussed.
\end{abstract}

Keywords: collaborative filtering, recommender systems, mobile computing.

\section{Introdução}

Todo o usuário busca por informação que possa auxiliá-lo na realização de suas tarefas e para isso busca geralmente recursos e tecnologias que possam auxiliá-lo. A Internet sem dúvida passa a ser uma das maiores tecnologias que permitem a busca por 
informação, porém mesmo com ferramentas de busca o usuário tende a despender muito tempo nesta busca. Visando facilitar este acesso pela informação surgem os Sistemas de Recomendação.

Os Sistemas de Recomendação têm por objetivo filtrar informações de acordo com o perfil de interesses dos usuários e assim recomendar itens que atendam as expectativas e necessidades dos usuários quanto a informações (Adomavicius, 2005) (Cazella e Reategui, 2005).

A Filtragem Colaborativa (FC) aplicada a Sistemas de recomendação constituise em uma das técnicas de maior sucesso na web no que tange a recomendação. Esta técnica baseia-se na chamada filtragem social (Shardanand e Maes, 1995) onde as opiniões de usuários "vizinhos" ao usuário-alvo da recomendação são fundamentais no cálculo da recomendação a ser feita. Esses ditos "vizinhos" são geralmente identificados através do uso de técnicas estatísticas que são aplicadas a um grupo de usuários que possuem um histórico de preferências similares ao usuário-alvo (Balabanovic et al, 1997), por exemplo, usuários que consumiram itens semelhantes ao usuário alvo e apresentaram avaliações semelhantes para estes itens.

A maior parte da pesquisa na área dos sistemas de recomendação reside na investigação sobre algoritmos para a recuperação de informação personalizada (Breese, Heckerman e Kadie, 1998); (Sarwar, Karypis, Konstan e Riedl, 2000); (Geyer-Schulz e Hahsler, 2002), com aplicações principalmente voltadas ao comércio. Neste trabalho, os sistemas de recomendação foram empregados na área de educação, com o objetivo de identificar e sugerir aos estudantes conteúdo relevante personalizado de acordo com as leituras e atividades sendo realizadas.

Este artigo apresenta um modelo de um Sistema de Recomendação baseado em Filtragem Colaborativa (FC) para ambientes móveis, e para descrever o modelo alguns conceitos relacionados a Sistemas de Recomendação são abordados. O modelo foi instanciado e aplicado na recomendação de artigos científicos em uma turma do curso de Engenharia da Computação. Experimentos foram realizados buscando verificar a melhora na qualidade da recomendação enviada aos alunos, propiciando maior facilidade no acesso a informação relevante para o estudo da disciplina, no caso do experimento: Banco de Dados.

O artigo está estruturado em seis seções distribuídas da seguinte forma. Na seção 2 são abordados os Sistemas de Recomendação, buscando referenciais teóricos que sustentem a aplicação de tais sistemas na área da educação. Na seção 3 é apresentada a arquitetura do modelo LOCAL, modelo que interage e utilizará o serviço disponibilizado pelo protótipo do modelo proposto neste artigo. Na seção 4 apresenta-se em detalhes o modelo RECMOBCOLABORATIVA resultado desta pesquisa. Na seção 5 são descritos os experimentos e resultados obtidos, bem como o protótipo do modelo que possibilita a avaliação das recomendações realizadas.

\section{Sistemas de Recomendação}

Ao longo dos anos, pesquisadores tanto da psicologia quanto da educação têm levantado evidências acerca da importância da socialização na construção do conhecimento (Vygostky, 1984); (Maturana, 1995). Sabe-se que a construção do conhecimento ocorre individualmente mas que a colaboração é capaz de levar a uma construção de conhecimento efetiva e significativa para todos os envolvidos (Piaget, 1973). A aprendizagem colaborativa ocorre como resultado do compartilhamento do conhecimento entre múltiplos participantes. Para tanto, aparece a tecnologia computacional que apóia e facilita este processo, respeitando as diferenças individuais e possibilitando a livre expressão em posicionamentos e troca de idéias. 
Os sistemas de recomendação são fundamentalmente colaborativos na medida em que permitem aos usuários compartilhar informações sobre materiais consultados/adquiridos, possibilitando também que registrem suas impressões sobre estes materiais. Os sistemas de recomendação têm tido um importante papel no ambiente web no que diz respeito a auxiliar um usuário no acesso a informação relevante ou na escolha de itens de seu interesse. Nesse contexto itens podem ser artigos científicos (Corrêa e Cazella, 2007); (Silva e Cazella, 2005), filmes (Schafer et al, 1999), músicas, livros, software ou produtos de consumo em geral.

Através de técnicas de análise de dados usadas pelos sistemas de recomendação, estes podem ajudar uma pessoa a encontrar os itens que melhor atendam suas necessidades (Adonie, 2005). Entre os trabalhos relacionados estão os seguintes: $\mathrm{Na}$ educação, Reategui et al (2006) propuseram o emprego dos sistemas de recomendação tanto para identificar conteúdo relevante para cada estudante, quanto para promover a interação entre estes. A abordagem empregada fazia uso de um modelo probabilístico específico para propor aos estudantes materiais relevantes de um curso de algoritmos. Já o trabalho de Huang (Huang et al, 2005) foca no uso dos sistemas de recomendação para supermercados, apoiando a venda de itens com baixa procura. Nakatomi (Nakatomi, 2005) faz uso de um sistema de recomendação junto à personalização do contexto para configurar parâmetros de impressão de documentos.

Nos Sistemas de Recomendação são utilizadas em geral uma das três técnicas de filtragem de informação citadas a seguir: filtragem baseada em conteúdo (FBC), filtragem colaborativa (FC), também conhecida como filtragem social e filtragem híbrida $(\mathrm{FH})$.

\subsection{Técnica de Filtragem Colaborativa}

A técnica de filtragem colaborativa foi escolhida para aplicação nesta pesquisa devido as vantagens que esta apresenta sobre as demais técnicas. Uma das vantagens da técnica de Filtragem Colaborativa segundo Herlocker (Herlocker et al, 2000)0 é que essa técnica possui um modelo conceitual de operação de fácil entendimento, possibilitando analisar itens a serem recomendados sem preocupar-se com o conteúdo destes itens e sim focando nas avaliações dos itens. A operação de um sistema de recomendação por filtragem colaborativa é similar a recomendação verbal de pessoa para pessoa (Webster et al, 2007). Os usuários são supridos de recomendação seguindo três etapas: 1) usuário fornece seu perfil de avaliações; 2) a FC identifica usuários com perfis similares (vizinhos); 3) as avaliações dos vizinhos são combinadas para se gerar as recomendações. Com isso, a percepção do usuário dos passos, relacionados anteriormente, afetará sua percepção sobre todo o sistema.

A seguir as etapas serão detalhadas: na Etapa 1 calcula-se o peso em relação a similaridade do usuário-alvo (aquele que deverá receber uma recomendação) e os demais usuários. Isso pode ser calculado aplicando, por exemplo, o coeficiente do Coseno ou o coeficiente de Pearson (Cazella e Reategui, 2005). Este último coeficiente será usado no modelo proposto por este artigo devido a melhor adequação e resultado (Shardanand e Maes, 1995); na Etapa 2 seleciona-se, a partir dos resultados do cálculo da similaridade, um subconjunto de usuários com maiores níveis de similaridade, que serão denominados vizinhos. Estes vizinhos serão considerados no cálculo da predição do item a ser recomendado; na Etapa 3 normaliza-se as avaliações fornecidas pelos usuários ao item em análise para recomendação e calcula-se a predição, ponderando-se as avaliações dos vizinhos com seus respectivos pesos de similaridade. 


\subsection{Cálculo do Coeficiente de Similaridade}

Para o cálculo da similaridade o modelo proposto adotou o coeficiente de Pearson, uma vez que o mesmo é amplamente utilizado em modelos e mede o grau de relacionamento entre duas variáveis, variando de -1 (ausência total de correlação) a 1 (forte correlação). O cálculo deste coeficiente é realizado conforme a equação 1 (Cazella e Reategui, 2005):

$$
\operatorname{corr}_{a b}=\frac{\sum_{i}\left(\mathrm{r}_{a i-} \overline{\mathrm{r}_{a}}\right)\left(\mathrm{r}_{b i-} \overline{\mathrm{r}_{b}}\right)}{\sqrt{\sum_{i}\left(\mathrm{r}_{a i-} \overline{\mathrm{r}_{a}}\right)^{2} \sum_{i}\left(\mathrm{r}_{b i-} \overline{\mathrm{r}_{b}}\right)^{2}}}
$$

Sendo o corr $_{a b}$ a correlação do usuário alvo a com um determinado usuário b; $\mathrm{r}_{a i}$ : é a avaliação que o usuário ativo a atribuiu para o item i; $\mathrm{r}_{b i}$ : é a avaliação que o usuário ativo $\mathrm{b}$ atribuiu para o item $\mathrm{i} ; \overline{\mathrm{r}_{a}}$ é a média de todas as avaliações do usuário ativo a, em comum com o usuário $b ; \bar{r}_{b}$ é a média de todas as avaliações do usuário ativo b, em comum com o usuário a.

\subsection{Predição}

A predição é feita independentemente do coeficiente utilizado no cálculo da similaridade, pois ela será gerada através de uma média ponderada das avaliações dos vizinhos que obtiveram um coeficiente de similaridade aceitável, ou seja, com limiar igual ou superior, por exemplo, a 0,3. A equação 2 é utilizada para o cálculo da predição (Cazella e Reategui, 2005).

$$
p_{a i}=\overline{r_{a}}+\frac{\left.\sum_{b=1}^{n}\left(r_{b i}-\overline{r_{b}}\right) * \operatorname{corr}_{a b}\right)}{\sum_{b=1}^{n}\left|\operatorname{corr}_{a b}\right|}
$$

Sendo $\operatorname{corr}_{a b}$ é a correlação do usuário alvo a com um determinado usuário b; $p_{a i}$ a predição de um item i para um usuário alvo a; $\overline{r_{a}}$ é a média de todas as avaliações do usuário alvo a aos itens que foram pontuados por todos os seus usuários similares; ra é a avaliação que o usuário ativo $b$ atribuiu para o item $i$; $\bar{b}$ é a média de todas as avaliações do usuário b, em comum com o usuário a.

\section{Modelo Local}

O modelo LOCAL (LOcation and Context Aware Learning) (Barbosa et al, 2008); (Barbosa et al, 2007) utiliza informações de localização e de contexto como auxílio ao processo de ensino e de aprendizagem e a sua estrutura. Neste modelo LOCAL um sistema de localização acompanha a mobilidade dos aprendizes e, baseado nas suas posições físicas, explora oportunidades educacionais. $\mathrm{O}$ modelo RECMOBCOLABORATIVA (seção 4) utiliza recursos da arquitetura do LOCAL para viabilizar algumas funcionalidades e fornece serviço para o LOCAL, por isso, a importância de se descrever um pouco deste outro modelo nesta seção.

O modelo LOCAL (Barbosa et al, 2008); (Barbosa et al, 2007) compõe-se de: Sistema de perfil de usuário; Sistema de localização; Assistente Pessoal; Repositório de objetos de aprendizagem; Sistema de envio de mensagens contextuais e Tutor.

\section{Modelo RECMOBCOLABORATIVA}

Nesta seção será descrito em detalhes o modelo proposto para recomendação de itens baseado em filtragem colaborativa aplicado a ambientes móveis. Na figura 1 é apresentado o modelo RECMOBCOLABORATIVA e o seu funcionamento é descrito a seguir. Deve-se observar que a figura descreve a integração do modelo LOCAL 
(primeira elipse da figura 1) descrito na seção 3 deste artigo e o modelo RECMOBCOLABORATIVA (segunda elipse da figura 1).

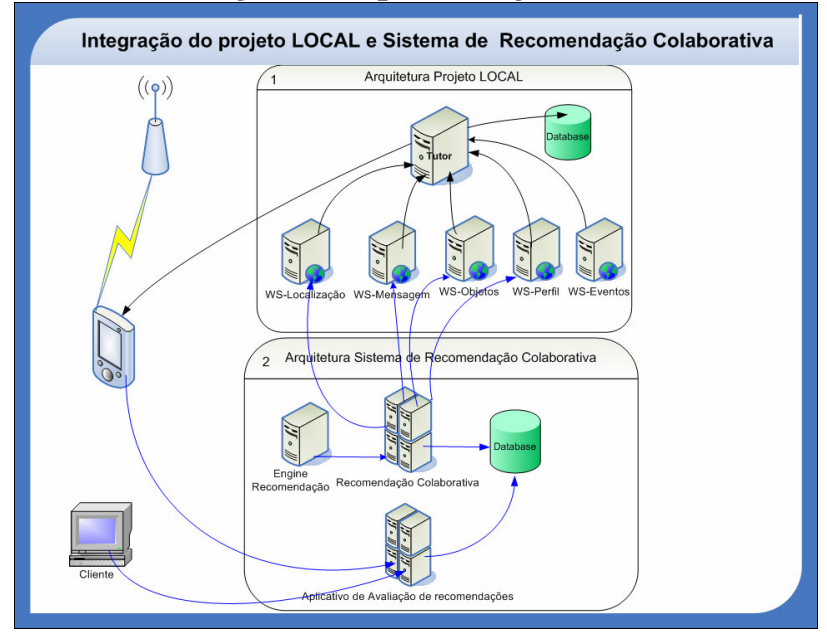

Figura 1. Modelo RECMOBCOLABORATIVA.

Conforme pode ser observado na figura 1, o modelo RECMOBCOLABORATIVA compõe-se de: Módulo de Recomendação - responsável pelo cálculo das similaridades e definição dos vizinhos próximos, bem como a predição de itens a serem recomendados; Recomendação Colaborativa - responsável pela interação com o projeto LOCAL e encaminhamento das recomendações produzidas para que o LOCAL encaminhe as mesmas para os dispositivos móveis; Aplicativo de Avaliação de recomendações - aplicativo voltado a coleta das avaliações dos usuários aos itens recomendados; Base de dados - com os itens a serem recomendados, os usuários cadastrados e seus perfis, bem como as avaliações fornecidas pelos usuários. O modelo descrito foi implementado através de um protótipo e seu funcionamento é descrito a seguir, apresentando a interação com a arquitetura do Local: 1) $\mathrm{O}$ tutor de recomendação (Módulo de recomendação) é executado como um serviço no servidor do projeto LOCAL, e este serviço é executado diariamente ao final do dia em busca de recomendações para os usuários; 2) A primeira vez em que este serviço for executado, o protótipo irá calcular o coeficiente de similaridade entre todos os usuários cadastrados no sistema (conforme descrito na seção 2.1.1) e depois irá calcular a predição (conforme descrito na seção 2.1.2) para todos os itens passíveis de recomendação disponíveis no sistema (no caso do experimento realizado e descrito na seção 5 deste artigo, estes itens são artigos científicos). Após esta primeira execução os dados referentes aos coeficientes de similaridade dos usuários e predições são persistidos na base de dados (database); 3) Nas execuções subseqüentes do protótipo são realizados novos cálculos caso existam novos usuários cadastrados, novos itens, e novas avaliações de itens. Sendo assim, o protótipo verificará se novas avaliações de artigos pelos usuários foram persistidas no banco de dados (database) e a partir destas novas avaliações, o protótipo irá recalcular o coeficiente de similaridade apenas para os usuários envolvidos nessas novas avaliações e finalmente calculará a predição de itens para os usuários; 4) Após a execução dos cálculos, tanto de coeficiente de similaridade quanto os valores de predição, o protótipo verificará se alguma predição de item foi feita para algum usuário, em caso positivo, o protótipo envia ao tutor do LOCAL a recomendação do item destinada ao usuário por meio de um Web Service; 5) O tutor do LOCAL receberá as recomendações, resultantes da filtragem colaborativa do protótipo que implementa o modelo RECMOBCOLABORATIVA, e após o usuário-alvo da recomendação se 
conectar ao ambiente do projeto LOCAL, ele receberá em seu dispositivo móvel todas as recomendações disponíveis para o usuário-alvo.

O usuário ao se conectar e se identificar ao sistema usando um dispositivo móvel (palm $H P$ iPAQ), receberá automaticamente todas as recomendações calculadas pelo protótipo que implementa o modelo RECMOBCOLABORATIVA.

\section{Experimentos e Resultados}

Objetivando avaliar o modelo RECMOBCOLABORATIVA através do protótipo implementado foram realizados dois experimentos com alunos do curso de graduação em Engenharia da Computação da Unisinos ${ }^{1}$ (Universidade do Vale do Rio dos Sinos) na disciplina de Banco de Dados.

\subsection{Método de Pesquisa}

O método de pesquisa aplicado neste trabalho foi o de experimentação visando avaliar o modelo através do uso de seu protótipo implementado junto a uma amostra de acadêmicos. Esta amostra foi obtida por conveniência, constituindo-se em uma amostra não probabilística, sendo assim deve-se observar que os resultados obtidos com intuito de avaliar o modelo não são generalizáveis. A amostra foi constituída por 11 discentes formandos do curso de bacharelado em Engenharia da Computação da Unisinos. Para viabilizar a avaliação do modelo implementado foi gerada uma base sintética de artigos científicos relacionados a disciplina de banco de dados que se configuram como os itens a serem futuramente recomendados aos amostrados.

O experimento constitui-se em identificar os usuários com similaridade de "gostos" pelos itens e predizer quais novos itens deveriam ser recomendados para estes usuários utilizando para isso dispositivos móveis e a técnica de recomendação colaborativa implementada no protótipo. Os usuários ao receberem a recomendação foram convidados a avaliarem os itens recomendados, utilizando uma escala likert de 5 pontos, onde o valor 1 representa ausência de satisfação com a recomendação recebida e 5 representa total satisfação com a recomendação recebida.

\subsection{Métricas de Avaliação}

Com o objetivo de avaliar o modelo se fez necessário a escolha de uma métrica que possibilitasse comparar a avaliação fornecida por um usuário a um determinado item e a predição feita pelo protótipo. Para tanto, foi adotada a métrica denominada MAE (Mean Absolute Error) e precisão.

Com estas métricas é possível verificar qual a precisão das recomendações feitas com base no modelo implementado no que se refere à margem de erro da predição. $\mathrm{O}$ valor MAE para cada predição deve ser minimizado buscando uma recomendação mais adequada. Mais formalmente o MAE pode ser descrito como: se $\left\{r_{1}, \ldots, r_{N}\right\}$ são todos os valores reais dentro do conjunto de avaliações atribuídas por um usuário a um determinado item, e $\left\{p_{1}, \ldots, p_{N}\right\}$ são os valores das predições das avaliações atribuídas por um usuário a este mesmo item, então o MAE constitui-se no quociente dos valores dos erros entre estas avaliações somados, $\mathrm{E}=\{1, \ldots, N\}=\left\{p_{1}-r_{1}, \ldots, p_{N}-r_{N}\right\}$, segundo a equação 3:

$$
|\bar{E}|=\frac{\sum_{l=1}^{N}|E|}{N}
$$

Para calcular o valor do MAE foi necessário calcular através do protótipo a avaliação que o usuário possivelmente daria ao artigo a ser recomendado (predição), e

${ }^{1}$ www.unisinos.br 
depois da recomendação realmente feita, o usuário-alvo foi convidado a avaliar o artigo. Com base na diferença destes dois valores referentes a avaliação (avaliação predita e avaliação real) o MAE é calculado e seu resultado constituindo-se no erro da predição.

No contexto de sistemas de recomendação, a precisão é a relação entre a nota dada pelo usuário a um item que lhe foi recomendado e a nota da predição. Assumiu-se neste experimento que os artigos recomendados aos usuários são aqueles que possuem valor de predição igual ou superior a 3 pontos (em uma escala likert de 5 pontos). Com o uso desta métrica, foi possível verificar qual o índice de acerto do modelo implementado.

\subsection{Primeiro experimento e resultados}

O primeiro experimento teve como objetivo coletar as primeiras avaliações dos amostrados para possibilitar a aplicação da filtragem colaborativa, uma vez que não existiam avaliações para possibilitar o cálculo da predição. Este experimento também teve por objetivo lidar com uma das limitações da técnica de filtragem colaborativa, o problema do novo usuário (Schafer, 1999). Conforme informado, inicialmente foi selecionada uma base inicial de artigos científicos para recomendar aos usuários amostrados. Foram selecionados um total de 30 artigos científicos e estes foram distribuídos para os amostrados do curso de Engenharia da Computação conforme interesses de cada um destes alunos (a distribuição inicial de artigos foi realizada com o auxílio do professor da disciplina que estes alunos amostrados cursavam).

Os acadêmicos da amostra receberam inicialmente a recomendação de alguns artigos para que avaliassem os mesmos. A pontuação foi feita utilizando para isso uma ferramenta de avaliação de artigos, parte do protótipo. Em média foram atribuídos 9 artigos por aluno. Estas avaliações iniciais ajudaram o modelo implementado a identificar as preferências de cada usuário e também auxiliou na viabilização do cálculo do coeficiente de Pearson e cálculo futuro da predição e recomendação (segundo experimento). Após a avaliação realizada uma matriz de avaliações iniciais foi gerada com um total de 107 avaliações. Para as avaliações na interface a escala likert de 5 pontos foi adaptada para conceitos, desta forma a avaliação com valor 1 correspondeu ao conceito péssimo, e a avaliação com valor 5 correspondeu ao conceito excelente.

Como resultados deste primeiro experimento pode-se afirmar que o percentual de usuários que realmente participaram e avaliaram os artigos ficou em 72,72\%, e o problema do novo usuário foi minimizado. Com estas avaliações iniciais foi possível dar início as recomendações colaborativas.

De posse dos primeiros artigos avaliados o modelo implementado pôde calcular a similaridade entre os usuários que participaram das avaliações, usando-se para isso o cálculo do coeficiente de Pearson (subseção 2.2). Quanto a distribuição das similaridades calculadas entre os usuários amostrados do experimento observou-se que $55,81 \%$ das correlações calculadas entre os usuários amostrados são correlações fortes, $9,30 \%$ são correlações fracas e $34,88 \%$ delas não se pode afirmar nada quanto a similaridade entre estes usuários. Após serem calculadas as similaridades entre os usuários foi possível iniciar o cálculo das predições dos artigos a serem recomendados para os usuários, dando início ao segundo experimento.

\subsection{Segundo Experimento e resultados}

O segundo experimento consistiu-se na geração de recomendações efetivas baseadas nas avaliações iniciais produzidas pelos amostrados. Neste o modelo implementado calculou a predição de artigos e o protótipo encaminhou estas recomendações para os usuários em seus dispositivos móveis.

O modelo implementado, recomendou apenas artigos não acessados por estes amostrados no primeiro experimento e que no cálculo da predição obtiveram nota de 
predição igual ou superior a valor 3 dentro de uma escala likert de 5 pontos. Deve ser destacado que não foram geradas recomendações para usuários que obtiveram um índice de similaridade menor que 0,3 com outros usuários.

Uma vez que as recomendações foram calculadas pelo modelo implementado no protótipo, os amostrados foram convidados a avaliarem estes artigos utilizando a interface da aplicação (figura 2).

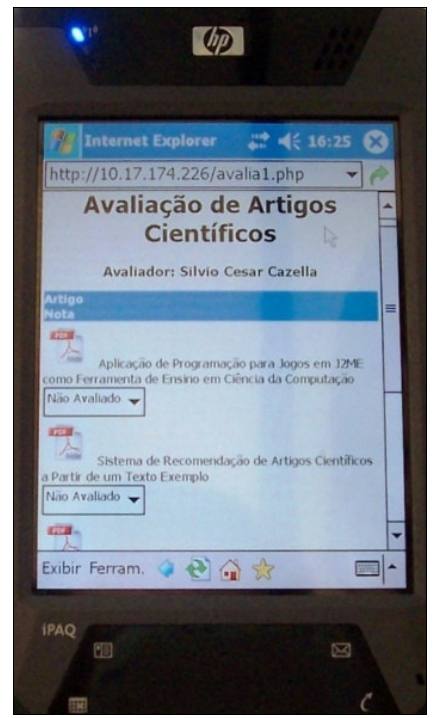

Figura 2. Interface do aplicativo para avaliação das recomendações.

A Tabela 1 apresenta os resultados obtidos neste experimento. Conforme podese observar nesta tabela, o modelo implementado não gerou recomendações para os usuários $U_{1}, U_{5}, U_{8}, U_{10}$ e $U_{11}$. Estes usuários não receberam recomendações devido a alguns não possuírem vizinhos com índice de similaridade maior do que 0,3 ou pelo fato de não terem participado da primeira rodada de avaliações do experimento primeiro, não possibilitando desta forma, realizar o cálculo do coeficiente de Pearson para estes usuários.

Após as predições terem sido calculadas e as avaliações terem sido fornecidas pelos amostrados o MAE foi calculado. Com base no resultado do MAE, observou-se uma diferença média entre as predições feitas pelo modelo implementado e as avaliações feitas pelos usuários de 0,76 pontos. Desta forma a precisão das predições geradas pelo modelo implementado foi de $79,75 \%$.

Na Tabela 1, são apresentados o usuário, o código do artigo que lhe foi recomendado, o valor de predição calculado pelo modelo implementado (este valor de predição representa o valor que se espera que o usuário amostrado atribua ao artigo recomendado após a leitura do mesmo), o valor da avaliação real do usuário (este valor representa a avaliação real que o usuário amostrado atribuiu ao artigo recomendado após a leitura ter sido realizada), o erro absoluto calculado entre as duas avaliações (MAE) e a precisão da predição feita pelo modelo implementado.

Tabela 1. Resultados do segundo experimento

\begin{tabular}{|c|c|c|c|c|c|}
\hline Usuário & Artigo & Predição & Avaliação & MAE & Precisão \\
\hline $\mathrm{U}_{2}$ & 18 & 3 & 2 & 1,04 & $65,79 \%$ \\
\hline $\mathrm{U}_{3}$ & 25 & 3 & 4 & 0,61 & $82,01 \%$ \\
\hline $\mathrm{U}_{4}$ & 10 & 4 & 3 & 1,59 & $65,36 \%$ \\
\hline $\mathrm{U}_{4}$ & 16 & 3 & 3 & 0,00 & $100,00 \%$ \\
\hline
\end{tabular}




\begin{tabular}{|c|c|c|c|c|c|}
\hline Usuário & Artigo & Predição & Avaliação & MAE & Precisão \\
\hline $\mathrm{U}_{6}$ & 10 & 4 & 3 & 1,00 & $75,00 \%$ \\
\hline $\mathrm{U}_{6}$ & 22 & 3 & 3 & 0,55 & $84,51 \%$ \\
\hline $\mathrm{U}_{7}$ & 22 & 3 & 2 & 1,75 & $53,33 \%$ \\
\hline $\mathrm{U}_{9}$ & 10 & 4 & 4 & 0,36 & $91,74 \%$ \\
\hline $\mathrm{U}_{9}$ & 16 & 3 & 3 & 0,00 & $100,00 \%$ \\
\hline Média & -- & -- & -- & $\mathbf{0 , 7 6}$ & $\mathbf{7 9 , 7 5 \%}$ \\
\hline
\end{tabular}

\section{Conclusão}

Dentro do contexto de aprendizagem com mobilidade foi apresentado neste trabalho um modelo de um sistema de recomendação baseado em filtragem colaborativa que recomenda itens (artigos científicos) para usuários através do uso de dispositivos móveis. O principal objetivo do modelo proposto é possibilitar que um usuário possa receber uma recomendação relevante para seu aprendizado a qualquer momento em que inicie o uso do sistema e em qualquer lugar devido aos dispositivos móveis.

A maior contribuição deste trabalho foi agregar ao modelo LOCAL um maior grau de qualidade nas recomendações geradas para os usuários. A predição das recomendações é baseada na visão da comunidade de usuários do sistema. A técnica de filtragem colaborativa é transparente aos usuários, no sentido de que eles têm ciência de como aquela recomendação é gerada, sendo assim, o usuário torna-se um colaborador do sistema e não somente um consumidor do conteúdo gerado por ele. A livre recomendação de conteúdo personalizado aos estudantes, deixando a estes a iniciativa de decidirem os materiais que serão efetivamente consultados, dá suporte às teorias de aprendizagem que enfatizam a importância da autonomia dos aprendizes (Piaget 1973). Conforme a precisão obtida nos experimentos utilizando o modelo proposto e implementado, que foi de 79,75\% na amostragem, demonstrou-se que o mesmo atingiu um resultado positivo quanto a predição. Com isso o modelo conseguiu atingir seus objetivos para essa amostra por conveniência, agregando qualidade às recomendações feitas para os amostrados, ao mesmo tempo em que essas recomendações eram enviadas a seus dispositivos móveis através da integração do modelo RECMOBCOLABORATIVA com o modelo LOCAL.

Como trabalhos futuros de pesquisa a serem realizados, o modelo RECMOBCOLABORATIVA poderia ser estendido para incluir a relevância de opinião de um usuário para auxiliar no processo de recomendação colaborativa. Outro trabalho futuro refere-se ao estudo de redes sociais entre aqueles usuários que possuem índice de similaridade dentro da escala de interesse e para lidar com o problema da esparcialidade, poderia ser aplicada uma técnica de redução de dimensões sobre a base de dados.

\section{Referências}

Adomavicius, G., Tuzhilin A., Toward the Next Generation of Recommender Systems: A Survey of the State of-the-Art and Possible Extensions, IEEE Transactions on Knowledge and Data Engineering, 17, 6 (Jun. 2005), 734-749.

Adonie, R., Russo, J. E., Dean, R., Crossing the rubicon for an intelligent advisor. In: Workshop Beyond Personalization, 2005, San Diego, CA, USA, 7-12.

Balabanovic, M., Shoham, Y. Fab: Content-Based, Collaborative Recommendation. Communications of the ACM, 40, 3 (Mar. 1997), 66-72. 
Barbosa, J. L. V. , Hahn, R.,; Rabello, S. A, Barbosa, D. N. F. . LOCAL: a Model Geared Towards Ubiquitous Learning. In: 39th ACM Technical Symposium on Computer Science Education (SIGCSE), 2008, Portland. Proceedings of the ACM SIGCSE 2008. New York : ACM Press, 2008. 432-436.

Barbosa, J., Hahn, R., Rabello, S., Barbosa, D., Distribuição de Conteúdo em Ambientes Conscientes de Contexto. In: XIII Simpósio Brasileiro de Sistemas Multimídia e Web, 2007, Gramado.

Breese, J., Heckerman, D. \& Kadie, C. Empirical Analysis of Predictive Algorithms for Collaborative Filtering. In: Proceedings of the Fourteenth Conference on Uncertainty in Artificial Intelligence, Madison, WI, July, Morgan Kaufmann. 1998. Cazella, S. C., Reategui, E., Mini-course: Recommender Systems. In: Encontro Nacional de Inteligência Artificial (ENIA), 2005, São Leopoldo, RG, Brazil.

Corrêa, I., Cazella, S. C., Um modelo para recomendação de itens baseado em filtragem colaborativa para dispositivos móveis. In: Workshop on undergraduate research, 2007, Gramado. Webmedia.

Geyer-Schulz, A. \& Hahsler, M. Evaluation of Recommender Algorithms for an Internet Information Broker based on Simple Association Rules and on the RepeatBuying Theory. Proceedings WEBKDD'2002, Eds. B. Masand, M. Spiliopoulou, J. Srivastava and O. Zaïane. Edmonton, Canada, pp.100-114. July 2002.

Herlocker, J., Konstan, J., Riedl, J., Explaining Collaborative Filtering Recommendations. In: Proceedings of the 2000 ACM conference on Computer supported cooperative work, 241-250.

Huang, H., Lin, K., Hsu, J. Y., Hsu, C., Item-triggered recommendation for identifying potential customers of cold sellers in supermarkets. In: Workshop Beyond personalization, 2005, San Diego, CA, USA, 37-42.

Maturana, H. and Varela, F. Árvore do conhecimento. Ed. Psy, Campinas, 1995. Nakatomi, M., What affects printing options? - Toward personalization \& recommendation system for printing devices. In: Workshop Beyond personalization, 2005, San Diego, CA, USA, 60- 65.

Piaget, J. Estudos Sociológicos. Rio de Janeiro: Forense. 243p. 1973. Reategui, E.; Boff, E.; Ceron, R. F.; Viccari, R. M. Um Agente Animado para Ambientes de Aprendizagem Colaborativos. Revista Brasileira de Informática na Educação, Porto Alegre, RS, v. 14, n. 3, p. 27-38, 2006.

Sarwar, B., Karypis, G., Konstan, J. \& Riedl, J. Analysis of recommender algorithms for e-commerce. In Proceedings of the 2nd ACM Conference on Electronic Commerce, pp.158-167, 2000.

Schafer, J. B. et al. Recommender system in Ecommerce, In: Proceedings of the ACM Conference on Electronic Commerce (EC' 99), N. Y., USA, 158-166.

Shardanand, U., Maes, P., Social information filtering: Algorithms for automating "word of mouth", In: Proceedings of Human Factors in Computing Systems (CHI '95), Denver, Colorado, USA.

Silva F. W., Cazella, S. C., STAR: Um Framework para recomendação de artigos científicos baseado na relevância da opinião dos usuários e em filtragem colaborativa. In: Encontro Nacional de Inteligência Artificial (ENIA), 2005, São Leopoldo, RG, Brazil. 


Co carmans

Webster, A. and Vassileva, J. Push-Poll Recommender System: Supporting Word of Mouth. In: Proceedings of User Modeling (UM 2007), Springer-Verlag, Berlin, 2007. 